This item was submitted to Loughborough's Research Repository by the author.

Items in Figshare are protected by copyright, with all rights reserved, unless otherwise indicated.

\title{
Sleep-related crash characteristics: Implications for applying a fatigue definition to crash reports
}

PLEASE CITE THE PUBLISHED VERSION

http://dx.doi.org/10.1016/j.aap.2015.11.024

PUBLISHER

(c) Elsevier

VERSION

AM (Accepted Manuscript)

\section{PUBLISHER STATEMENT}

This work is made available according to the conditions of the Creative Commons Attribution-NonCommercialNoDerivatives 4.0 International (CC BY-NC-ND 4.0) licence. Full details of this licence are available at: https://creativecommons.org/licenses/by-nc-nd/4.0/

\section{LICENCE}

CC BY-NC-ND 4.0

\section{REPOSITORY RECORD}

Filtness, Ashleigh J., Kerry A. Armstrong, Angela Watson, and Simon S. Smith. 2019. "Sleep-related Crash Characteristics: Implications for Applying a Fatigue Definition to Crash Reports". figshare.

https://hdl.handle.net/2134/20393. 
1 Sleep-related crash characteristics: Implications for applying a fatigue

2 definition to crash reports

3

4

5 AJ Filtness*, K Armstrong, A Watson, SS Smith

6

7 Queensland University of Technology (QUT), Centre for Accident Research and Road Safety -

8 Queensland (CARRS-Q)

$9 *$ * Corresponding author:

10 Ashleigh Filtness, Ph.D.

11 CARRS-Q, Queensland University of Technology, K block, 130 Victoria Park Road, Kelvin Grove,

12 Queensland, 4059, Australia

13 Tel: +61731387713

$14 \quad$ Fax: +61731380111

15 Email: Ashleigh.filtness@qut.edu.au

16 


\section{Abstract}

2 Sleep-related (SR) crashes are an endemic problem the world over. However, police officers report

3 difficulties in identifying sleepiness as a crash contributing factor. One approach to improving the

4 sensitivity of SR crash identification is by applying a proxy definition post-hoc to crash reports. To

5 identify the prominent characteristics of SR crashes and highlight the influence of proxy definitions,

6 ten years of Queensland (Australia) police reports of crashes occurring in $\geq 100 \mathrm{~km} / \mathrm{h}$ speed zones

7 were analysed. In Queensland, two approaches are routinely taken to identifying SR crashes. First,

8 attending police officers identify crash causal factors; one possible option is 'fatigue/fell asleep'.

9 Second, a proxy definition is applied to all crash reports. Those meeting the definition are

10 considered SR and added to the police-reported SR crashes. Of the 65,204 vehicle operators involved

11 in crashes 3,449 were police-reported as SR. Analyses of these data found that male drivers aged 16-

1224 years within the first two years of unsupervised driving were most likely to have a SR crash.

13 Collision with a stationary object was more likely in SR than in not-SR crashes. Using the proxy definition 9,739 (14.9\%) crashes were classified as SR. Using the proxy definition removes the findings that SR crashes are more likely to involve males and be of high severity. Additionally, proxy defined SR crashes are no less likely at intersections than not-SR crashes. When interpreting crash data it is important to understand the implications of SR identification because strategies aimed at reducing the road toll are informed by such data. Without the correct interpretation, funding could be misdirected. Improving sleepiness identification should be a priority in terms of both improvement to police and proxy reporting.

Key words: driver sleepiness, driver drowsiness, driver fatigue, proxy definition, surrogate fatigue measures 


\section{$1 \quad 1 \quad$ Introduction}

2 Every year driver fatigue or sleepiness is responsible for countless crashes the world over (Akerstedt

3 2000). Using a case controlled approach the population attributable risk for driving when sleepy is

4 reported to be $19 \%$, meaning that cessation of all driving when sleepy would result in a $19 \%$ decrease in the total number of crashes (Connor et al. 2002). The impact of such crashes for those involved can be devastating, as sleep-related (SR) crashes are highly likely to be fatal or to result in serious injury (Connor et al. 2002). However, there are several barriers faced by road safety authorities, each of which makes reducing the prevalence of driver sleepiness a difficult problem to address. First, when compared to other causes of driving impairment such as alcohol and drugs, SR impairment can be hard to identify. Enforcement officers report difficulty in identifying driver fatigue (Radun et al. 2013). This is influenced both by a lack of objective and reliable tests for sleepiness analogous to those available for alcohol or drugs (Pack et al. 1995), but also through deficiencies in officer training in the identification of SR crashes (Radun et al. 2013). The number of reported SR crashes is underestimated within crash data (Akerstedt 2000). Some road safety authorities address this issue by using proxy definitions of fatigue to supplement police reporting.

Proxy definitions are designed to be applied to all police-reported crashes as part of a post-hoc analysis to improve the sensitivity of SR crash identification. The Australian Transport Safety Bureau (ATSB) has developed a recommended proxy definition, and five of the eight Australian jurisdictions have incorporated their own proxy definition into standard practices. Within Queensland, Australia, the attending police officer is responsible for identifying the causes of any crash; as part of this process officers have the option of attributing one of the causes to 'fatigue/fell asleep'. There is currently no standard methodology for identifying 'fatigue/fell asleep' as a crash causal factor. All roadside police officers attend a basic crash investigation course as part of their training. In the event of a serious incident (fatal or serious injury) trained crash investigators from the Forensic Crash Investigation unit will attend. These officers specialise in identifying crash causal factors and will take steps beyond roadside investigation (e.g. checking shiftwork rosters, telephone records and 
1 bank statements) to identify how long a person has been awake and driving for, if they suspect

2 fatigue. To supplement this, the Queensland proxy definition is applied post-hoc by the State

3 Government Department of Transport and Main Roads. This definition states that sleepiness is a

4 contributing factor in all single-vehicle crashes in $\geq 100 \mathrm{~km} / \mathrm{h}$ speed zones which occur between $2 \mathrm{pm}$

5 to $4 \mathrm{pm}$ and $10 \mathrm{pm}$ to $6 \mathrm{am}$, or where a vehicle leaves the roadway with the driver not attempting to

6 avoid the crash. The process of applying a proxy definition is a desk-based exercise outside of the

7 remit of the police. When reporting fatigue crashes road safety authorities either use police-

8 reported SR crashes alone or use proxy identified in addition to the police-reported SR crashes.

9 The content of proxy definitions have some grounding in scientific research (e.g. single vehicle run

10 off the road crashes are most likely to be SR (Horne and Reyner 1995b, Pack et al. 2006)). However,

11 there has been some criticism that proxy definitions are too narrow (Crummy et al. 2008) and that they only capture a specific subset of SR crashes (Armstrong et al. 2013).

Despite their limitations, proxy definitions are being actively used by road safety authorities so it is vital to understand the implications this has on crash statistics. This is particularly important when it is considered that road safety authorities will use crash statistics from their own jurisdictions to inform future investment in road safety. In deciding whether to invest in measures to reduce driver

17 fatigue, road safety authorities are likely to first consider if SR crashes are prevalent in their

18 jurisdiction and second to identify which drivers are most at risk. With such crucial decisions being

19 made based on region-specific data, the implications of how the data is processed and interpreted

20 should be fully understood.

21 The aim of this paper is twofold: First, to characterise SR crashes in high speed zones $(\geq 100 \mathrm{~km} / \mathrm{h})$ and the vehicle operators involved in them. Second, to demonstrate the implications of using proxy definitions for estimates of the prevalence and characteristics of SR crashes. 


\section{Method}

\subsection{Crash data}

Queensland police-reported crashes of motorised vehicles occurring in 'high' ( $\geq 100 \mathrm{~km} / \mathrm{h}$ ) speed zones between $1^{\text {st }}$ January 2000 and $31^{\text {st }}$ December 2009 were examined. Ten years of crash data provides sufficient scope for identifying general trends, and permits meaningful comparisons between SR and not sleep-related crashes (not-SR). Police crash reports detail all police attended crashes which occur on a public road, and where any of: a person was killed or injured, a vehicle towed, or greater than $\$ 2500$ of damage to property other than vehicles was incurred. Crashes resulting from medical conditions and deliberate acts are excluded. Police crash reports detail vehicle operator characteristics and crash characteristics, as well as crash causal factors identified by the reporting Queensland Police Services (QPS) officer.

\subsection{Sleep-related classification}

Three analysis approaches were taken to the classification of SR crashes.

First, all crash reports where the contributory factor "fatigue/fell asleep" was noted by the police officer were classified as SR. The remainder of the crash reports where classified as not-SR.

Second, all crash reports regardless of police officer classification were subject to the Queensland proxy definition of fatigue. Following the Queensland proxy definition, all crashes in $\geq 100 \mathrm{~km} / \mathrm{h}$ zones which occur between $2 \mathrm{pm}$ to $4 \mathrm{pm}$ and $10 \mathrm{pm}$ to $6 \mathrm{am}$, in which a single vehicle was involved, or where the vehicle leaves the roadway with the driver not attempting to avoid the crash, were classified as SR. The remainder of the crash reports where considered as not-SR.

Finally, all crash reports with either a police or proxy classification of SR were considered to be SR. The remainder of the crash reports where considered as not-SR. This approach is commonly taken by road authorities who use proxy definitions. 


\subsection{Statistical analysis}

Examination of vehicle operator and crash characteristics was undertaken to identify any significant differences between SR and not-SR crashes. A range of characteristics were considered, those most interesting in terms of differences between SR and not-SR are reported in the current paper. Characteristics not presented include: vehicle type, number of occupants, lighting conditions, atmospheric conditions and road surface conditions. Categorical data were analysed using Chisquare ( $\chi 2)$ tests with Cramer's V ( $\phi c)$ as an estimate of effect size. As suggested by Aron (2012), a Cramer's $V$ of less than .10 was considered to be a small effect size, between .10 and .30 moderate, and more than 0.30 a large effect size. Analysis was undertaken three times following three different methods of identify SR crashes: (1) police-reported; (2) proxy definition; and, (3) combination of police-reported and proxy definition.

A significance level $(\alpha)$ of .001 was used due to the use of multiple tests and a large sample size. Accordingly, adjusted standardised residuals outside \pm 3.29 were considered significant. In order to include vehicle operator characteristics as variables of interest, the main unit of analysis was the drivers/riders involved in crashes during the period, rather than crashes per se. All statistical analyses were conducted using IBM SPSS 19.0 statistical software.

\section{Results}

Overall, there were 65,204 vehicle operators (drivers and riders) involved in crashes on high speed ( $\geq$ $100 \mathrm{~km} / \mathrm{h}$ ) Queensland roads during the 10 year period of interest. Using police report alone, 3,449 (5.3\%) crashes were classified as SR. Using the proxy definition alone 9,739 (14.9\%) crashes were classified SR. When police-reported and proxy definition were combined, a total of $11,477(17.6 \%)$ crashes were classified as SR. Half $(n=1,738)$ of the police-reported crashes did not meet the proxy definition. 


\begin{tabular}{lrrr}
\hline Variable & $\begin{array}{r}\text { Sleep- } \\
\text { related }\end{array}$ & $\begin{array}{r}\text { Not sleep- } \\
\text { related }\end{array}$ & \\
\hline Vehicle operator characteristics & & & \\
$\quad$ Male gender & $76.8 \%$ & $69.4 \%$ & $\chi^{2}(1)=81.44, \mathrm{p}<.001, \phi_{c}=.04$ \\
Aged 16-24 & $34.5 \%$ & $25.9 \%$ & $\chi^{2}(2)=128.23, \mathrm{p}<.001, \phi_{c}=.05$ \\
Provisional licence & $22.2 \%$ & $14.8 \%$ & $\chi^{2}(3)=165.49, \mathrm{p}<.001, \phi_{c}=.05$ \\
Illegal BAC & $7.7 \%$ & $4.5 \%$ & $\chi^{2}(1)=89.66, \mathrm{p}<.001, \phi_{c}=.04$ \\
Crash characteristics & & & \\
$\quad$ Severity (fatality/hospitalisation) & $43.5 \%$ & $31.8 \%$ & $\chi^{2}(4)=227.64, \mathrm{p}<.001, \phi_{c}=.06$ \\
$\quad$ Nature - Hit stationary object & $56.7 \%$ & $26.6 \%$ & $\chi^{2}(1)=1455.82, \mathrm{p}<.001, \phi_{c}=.15$ \\
$\quad$ Nature - overturned & $24.4 \%$ & $14.4 \%$ & $\chi^{2}(1)=297.68, \mathrm{p}<.001, \phi_{c}=.07$ \\
Intersection & $3.5 \%$ & $14.9 \%$ & $\chi^{2}(1)=329.48, \mathrm{p}<.001, \phi_{c}=.07$ \\
Traffic control & $0.6 \%$ & $5.1 \%$ & $\chi^{2}(1)=136.97, \mathrm{p}<.001, \phi_{c}=.05$ \\
Single vehicle & $81.4 \%$ & $46.0 \%$ & $\chi^{2}(1)=1511.10, \mathrm{p}<.001, \phi_{c}=.15$ \\
Time of day (10pm to 6am) & $38.9 \%$ & $12.3 \%$ & $\chi^{2}(1)=3197, \mathrm{p}<.001, \phi_{c}=.15$ \\
Time of day (2pm - 4pm) & $13.7 \%$ & $14.7 \%$ & $\chi^{2}(1)=3.35, \mathrm{p}=.067, \phi_{c}=.01$ \\
Day of week (weekend) & $36.8 \%$ & $27.1 \%$ & $\chi^{2}(1)=146.50, \mathrm{p}<.001, \phi_{c}=.05$ \\
\hline
\end{tabular}

\subsection{Proxy identified sleep-related crash characteristics}

The proportion of proxy definition identified SR crashes including each vehicle operator and crash characteristic is displayed in Table 2 . Involvement of single vehicle and the time of day are not 
1 prevalence of young (aged 16-24 years), provisional licence holders and illegal BAC involvement in SR

2 crashes. Furthermore, SR crashes were more likely to involve hitting a stationary object and for the

3 vehicle to overturn than not-SR crashes and to occur at the weekend. However, in contrast to police-

4 reported, there was no significant difference in the involvement of males and SR crashes were not

5 more severe than not-SR crashes. Additionally, using proxy definition the involvement of

$6 \quad$ intersections and traffic control were just as likely in SR as not-SR crashes.

7 


\begin{tabular}{lrrr}
\hline Variable & $\begin{array}{r}\text { Sleep- } \\
\text { related }\end{array}$ & $\begin{array}{r}\text { Not sleep- } \\
\text { related }\end{array}$ \\
\hline Vehicle operator characteristics & & & \\
Male gender & $78.5 \%$ & $75.4 \%$ & $\chi^{2}(1)=4.73, \mathrm{p}=.030, \phi_{c}=.04$ \\
Aged 16-24 & $38.1 \%$ & $31.5 \%$ & $\chi^{2}(2)=33.68, \mathrm{p}<.001, \phi_{c}=.10$ \\
Provisional licence & $25.5 \%$ & $19.6 \%$ & $\chi^{2}(3)=26.68, \mathrm{p}<.001, \phi_{c}=.09$ \\
Illegal BAC & $11.0 \%$ & $5.0 \%$ & $\chi^{2}(1)=43.32, \mathrm{p}<.001, \phi_{c}=.11$ \\
Crash characteristics & & & \\
Severity (fatality/hospitalisation) & $41.9 \%$ & $44.8 \%$ & $\chi^{2}(4)=6.46, p=.167, \phi_{c}=.04$ \\
Nature - Hit stationary object & $69.1 \%$ & $53.5 \%$ & $\chi^{2}(1)=178.23, p<.001, \phi_{c}=.23$ \\
Nature - overturned & $30.7 \%$ & $19.2 \%$ & $\chi^{2}(1)=60.48, p<.001, \phi_{c}=.13$ \\
Intersection & $3.3 \%$ & $3.7 \%$ & $\chi^{2}(1)=0.41, p=.521, \phi_{c}=.01$ \\
Traffic control & $0.1 \%$ & $1.0 \%$ & $\chi^{2}(1)=10.74, p=.001, \phi_{c}=.06$ \\
Single vehicle & $\mathrm{NA}$ & $\mathrm{NA}$ & \\
Time of day (10pm to 6am) & $\mathrm{NA}$ & $\mathrm{NA}$ & \\
Time of day (2pm - 4pm) & $\mathrm{NA}$ & $\mathrm{NA}$ & \\
Day of week (weekend) & $40.0 \%$ & $34.2 \%$ & $\chi^{2}(1)=12.58, \mathrm{p}<.001, \phi_{c}=.06$ \\
\hline
\end{tabular}

\subsection{Combined police-reported and proxy identified sleep-related crash characteristics}

5 Table 3 shows vehicle operator and crash characteristics when police-reported and proxy definition

6 are used in combination. Using this approach the same differences were observed as for police-

7 reported alone. SR crashes are significantly more likely to involve males, drivers aged 16-24,

8 provisional licences holders and illegal BAC. SR crashes were more likely to be serious, single vehicle,

9 involve hitting a stationary object and the vehicle overturning. They were more likely to occur at

10 weekends and between $10 \mathrm{pm}$ and $6 \mathrm{am}$ or $2 \mathrm{pm}-4 \mathrm{pm}$. Conversely, SR crashes were significantly less

11 likely to occur at intersections or areas with traffic control. There is some bias in this comparison as

12 time of day and single vehicle crashes are included in the proxy definition. 


\begin{tabular}{lrrr}
\hline Variable & $\begin{array}{r}\text { Sleep- } \\
\text { related }\end{array}$ & $\begin{array}{r}\text { Not sleep- } \\
\text { related }\end{array}$ & \\
\hline Vehicle operator characteristics & & & \\
Male gender & $74.8 \%$ & $68.7 \%$ & $\chi^{2}(1)=167.96, \mathrm{p}<.001, \phi_{c}=.05$ \\
Aged 16-24 & $33.9 \%$ & $24.7 \%$ & $\chi^{2}(2)=435.15, \mathrm{p}<.001, \phi_{c}=.08$ \\
Provisional licence & $20.5 \%$ & $14.1 \%$ & $\chi^{2}(3)=711.99, \mathrm{p}<.001, \phi_{c}=.11$ \\
Illegal BAC & $11.2 \%$ & $3.2 \%$ & $\chi^{2}(1)=1404.82, \mathrm{p}<.001, \phi_{c}=.15$ \\
Crash characteristics & & & \\
Severity (fatality/hospitalisation) & $37.4 \%$ & $31.3 \%$ & $\chi^{2}(4)=188.94, \mathrm{p}<.001, \phi_{c}=.05$ \\
Nature - Hit stationary object & $62.0 \%$ & $20.8 \%$ & $\chi^{2}(1)=8100.73, \mathrm{p}<.001, \phi_{c}=.35$ \\
Nature - overturned & $30.8 \%$ & $11.4 \%$ & $\chi^{2}(1)=2893.28, \mathrm{p}<.001, \phi_{c}=.21$ \\
Intersection & $5.8 \%$ & $16.2 \%$ & $\chi^{2}(1)=855.54, \mathrm{p}<.001, \phi_{c}=.12$ \\
Traffic control & $0.8 \%$ & $5.7 \%$ & $\chi^{2}(1)=506.54, \mathrm{p}<.001, \phi_{c}=.09$ \\
Single vehicle & $94.6 \%$ & $37.5 \%$ & $\chi^{2}(1)=12621.47, \mathrm{p}<.001, \phi_{c}=.44$ \\
Time of day (10pm to 6am) & $51.9 \%$ & $5.2 \%$ & $\chi^{2}(1)=19861, \mathrm{p}<.001, \phi_{c}=.38$ \\
Time of day (2pm-4pm) & $32.6 \%$ & $12.9 \%$ & $\chi^{2}(1)=3634.35, \mathrm{p}<.001, \phi_{c}=.16$ \\
Day of week (weekend) & $36.2 \%$ & $25.8 \%$ & $\chi^{2}(1)=527.22, \mathrm{p}<.001, \phi_{c}=.09$ \\
\hline
\end{tabular}

3

4

5

6

7

8

\section{Discussion}

The current work identified young (16-24 years of age), novice (within two years of licensure) drivers

to be over-represented in SR crashes in high speed zones $(\geq 100 \mathrm{~km} / \mathrm{h})$. This was the case for both

police-reported and proxy identification and is in line with previous research (Horne and Reyner

1995b, Pack et al. 1995, Connor et al. 2002, Sagaspe et al. 2010). Using three approaches to crash

data this paper demonstrates that a proxy definition can change the significance of crash

characteristics. In this case, analysis of data categorised by proxy definition found no significant

difference in the proportion of crashes that involved male drivers, nor differences in the severity of

SR compared to not-SR crashes. In contrast, both characteristics were more prevalent in SR crashes compared to not-SR crashes identified from police-reported fatigue. The use of a proxy definition also had implications for understanding the situational context of SR crashes. Specifically, road infrastructure features (intersections and traffic control) surrounding the crash were significantly 
1 less likely to be associated with SR than not-SR crashes in police-reported fatigue, whereas there was no difference between SR and not-SR in proxy identified fatigue.

Police-reported SR crashes were significantly more likely to be severe than not SR crashes, this

4 would be expected as case control comparison has demonstrated that SR crashes are more likely to be fatal or to result in serious injury (Connor et al. 2002). However, the same difference was not reported using the proxy identifed data. Serious crashes in Queensland are attended by the Forensic Crash Investigation unit who undertake a thorough investigation. This can include seeking additional information beyond that at the crash scene, such as details about the prior circumstances (e.g. previous sleep and shift work pattern). These additional details facilitate the Forensic Crash Investigation unit to make more informed decisions as to crash causation. In contrast the proxy definition is limited to information about the crash itself. It is possible that applying a proxy definition has greatest value for not severe crashes and is somewhat redundant in its application to severe crashes. Another noticeable difference between SR and not SR comparison for policereported and proxy identified was for male drivers. Previous research has reported that male drivers are overrepresented in SR crashes (e.g. Horne and Reyner 1995b). However, while this was the case for the police-reported data it was not for proxy identified. It is not clear why this difference occurred; it is possible that police may be expecting males to be overrepresented in SR crashes which may introduce bias in police reporting.

Within Queensland, attributed crash cause is not limited to a primary factor; this allows crash data analysis to consider implications for co-occurring causal factors. Regardless of analysis approach, illegal BAC was more often present in SR than in not-SR crashes. Driving simulator research has demonstrated that the combination of alcohol and sleepiness results in greater impairment than the additive effect of either alone (Horne et al. 2003, Banks et al. 2004). Specific consideration of combined sleep and alcohol related crashes may be warranted. Furthermore, driving simulator research has also demonstrated that sleepiness amplifies impairment from other crash causal 
1 factors e.g. distraction, (Anderson and Horne 2006). However, it is not clear how readily police will report fatigue in co-occurrence with other causal factors. Therefore, the genuine impact of sleep on road safety may be much greater than estimated here. Future research should consider police officer attitudes towards reporting fatigue as a co-occurring causal factor and characteristics of SR crashes co-occurring with another causal factor (e.g. illegal BAC) in comparison to those attributed to either factor in isolation. It could be considered that the current analysis provides some support for the proxy definition as two proxy features - single vehicle involvement and occurring between $10 \mathrm{pm}$ and $6 \mathrm{am}$ - were both more prevalent in SR crashes compared with not-SR crashes within the police-reported analysis. However, crashes between $2 \mathrm{pm}$ and $4 \mathrm{pm}$ are also included in the proxy definition and did not show a greater prevalence in the police-reported. Furthermore, while the police are not involved in applying the proxy definition it is possible that they may be aware of the common features of SR crashes identified from scientific literature. This may introduce bias if police judgment is not completely independent from the proxy definition. In both the police-reported and proxy analysis colliding with a stationary object was significantly more prevalent in SR than not-SR crashes and had one of the largest effect sizes. Collision with something in clear sight has previously been reported as a feature of SR crashes (Horne and Reyner 1995a) and is one of the most common outcomes of selfreported SR incidents (Armstrong et al. 2013). These findings provide some evidence for potential benefit of the inclusion of collision with a stationary object in proxy definitions, a feature which has also been reported by others (Crummy et al. 2008).

Police-reported SR crashes were significantly less likely to occur at intersections and on roads with traffic control. This finding is in line with previous studies reporting SR crashes as most prevalent on featureless roads (Horne and Reyner 1995b). However, there is no difference between SR and not-SR for either of these crash features within proxy identified data. It is possible that this discrepancy is caused by low specificity of the proxy definition. However, self-report of SR crashes and near-misses 
1 demonstrate that SR incidents are common in urban city areas (Sagaspe et al. 2010, Armstrong et al.

2 2013). Therefore, it is possible that police officers have low sensitivity in their reporting and are

3 failing to correctly identify sleepiness when crashes occur at intersections and areas of traffic

4 controls.

5 In lieu of an accurate objective measure to identify SR crashes increased investment in enhancing

6 both police and proxy SR crash identification is vital. In general the criteria within proxy definitions

7 are grounded in scientific evidence, but not all associated risk factors are considered. For example

8 one of the factors which substantially increases the risk of a SR crash is driving after five hours or less

9 sleep (Connor et al. 2002), and yet, this information is neither routinely collected by police nor

10 incorporated into proxy definitions. Furthermore, much of the research proxy definitions draw on

11 was not originally intended for the purpose of developing a proxy definition. Also, it has been shown that using SR criteria with police officers at crash scenes identifies a greater prevalence of SR crashes than the post-hoc application of proxy definition criteria to crash data (Horne and Reyner

14 1995b).

Currently, prevalence of police-reported SR crashes in Queensland is lower than would be expected from previous research (Horne and Reyner 1995b, Connor et al. 2002) providing strong evidence that police need assistance and/or specialised training in SR identification. Using a proxy definition increased the prevalence of SR crashes to $18 \%$. While this proportion is closer to that obtained from high-quality case control investigation (Connor et al. 2002) it is still an estimate of SR crashes. Approximately half of the police-reported SR crashes did not meet the proxy definition, suggesting that proxy definitions are only identifying a subset of SR crashes (Armstrong et al. 2013). The accuracy of proxy definitions is particularly uncertain as they do not closely reflect drivers subjective reports of SR crashes (Armstrong et al. 2013) and have been criticised for being too narrow (Crummy et al. 2008). 
1 It is acknowledged that findings are limited to crashes that were reported to the police. Additionally,

2 the ability of either the police or the proxy definitions to accurately identify fatigue accurately was

3 not validated. There is potential for inconsistency in reporting between individual police officers and particularly between those crashes attended by the Forensic Crash Investigation unit and those not. Serious crashes (fatal or serious injury) are attended by trained crash investigators. Furthermore, for serious crashes detailed information may be sought about the circumstances prior to the crash (e.g. prior sleep).The specialist training and additional information is likely to improve the ability to accurately recognise fatigue. In contrast, the Forensic Crash Investigation unit does not attend low severity crashes. As enforcement officers report difficulty in identifying fatigue as a crash casual factor (Radun et al. 2013) and without the additional training and information of the Forensic Crash Investigation unit it is possible fatigue identification in less serious crashes may not be as accurate. The data considered were only from Queensland Australia, it is unknown how comparable the findings are to other jurisdictions. Furthermore, analysis was limited to high speed crashes as this is a requirement of the proxy definitions. Future work may wish to consider if characteristics of SR crashes in slower speed zones are comparable to those found in high speed zones.

\subsection{Conclusion}

In conclusion, this study demonstrates that, regardless of police, proxy, or combined analysis techniques young drivers (aged 16-24) and provisional licence holders (within the first two years of licensure) are overrepresented in SR crashes compared to not-SR crashes. This group has the potential to benefit most from interventions to reduce driver sleepiness. Critically, the manner in which crash data are processed can influence both the prevalence and dominant characteristics attributed to SR crashes. The use of a proxy definition increases the proportion of all crashes identified as SR, however, there is a lack of tangible proxy definitions evaluations to verify their efficacy. In general proxy identified SR crashes have similar characteristics to police identified SR crashes, however, there are some subtle differences. Regardless of the limitations, proxy definitions are currently being used. Understanding this influence is important because strategies to reduce the 
road toll are informed by such data, without correct interpretation funding could be misdirected.

Improving sleepiness identification should be a priority in terms of both improvement to police and proxy reporting. Emphasis should be placed on both more accurate quantification of SR crashes but also development of interventions to limit the occurrence of SR crashes. There is a need for more accurate measurement of the true nature and extent of the role of sleepiness in crashes.

\section{Acknowledgements}

The authors wish to thank Queensland Department of Transport and Main Roads for providing the data.

\section{References}

Akerstedt, T., 2000. Consensus statement: Fatigue and accidents in transport operations. Journal of sleep research 9 (4), 395-395. DOI: 10.1046/j.1365-2869.2000.00228.x

Anderson, C., Horne, J., 2006. Sleepiness enhances distraction during a monotonous task. SLEEP 29 (4), 573.

Armstrong, K., Filtness, A.J., Watling, C.N., Barraclough, P., Haworth, N., 2013. Efficacy of proxy definitions for identification of fatigue/sleep-related crashes: An australian evaluation. Transportation Research Part F: Traffic Psychology and Behaviour 21, 242-252. DOI:10.1016/j.trf.2013.10.002

Aron, A. (2012). Statistics for Psychology 6th Edition: Pearson.

Banks, S., Catcheside, P., Lack, L., Grunstein, R.R., Mcevoy, R.D., 2004. Low levels of alcohol impair driving simulator performance and reduce perception of crash risk in partially sleep deprived subjects. SLEEP 27, 1063-1067.

Connor, J., Norton, R., Ameratunga, S., Robinson, E., Civil, I., Dunn, R., Bailey, J., Jackson, R., 2002. Driver sleepiness and risk of serious injury to car occupants: Population based case control study. Bmj 324 (7346), 1125. DOI: http://dx.doi.org/10.1136/bmj.324.7346.1125

Crummy, F., Cameron, P.A., Swann, P., Kossmann, T., Naughton, M., 2008. Prevalence of sleepiness in surviving drivers of motor vehicle collisions. Internal medicine journal 38 (10), 769-775. DOI: 10.1111/j.1445-5994.2008.01629.x

Horne, J., Reyner, L., 1995a. Driver sleepiness. Journal of sleep research 4 (s2), 23-29. DOI: 10.1111/j.1365-2869.1995.tb00222.x

Horne, J., Reyner, L., Barrett, P., 2003. Driving impairment due to sleepiness is exacerbated by low alcohol intake. Occupational and environmental medicine 60 (9), 689-692. DOI:10.1136/oem.60.9.689

Horne, J., Reyner, L.A., 1995b. Sleep related vehicle accidents. Bmj 310 (6979), 565-567. DOI: http://dx.doi.org/10.1136/bmj.310.6979.565

Pack, A.I., Maislin, G., Staley, B., Pack, F.M., Rogers, W.C., George, C.F., Dinges, D.F., 2006. Impaired performance in commercial drivers: Role of sleep apnea and short sleep duration. American 
journal of respiratory and critical care medicine 174 (4), 446-454.

DOI: $10.1164 / \mathrm{rccm} .200408-11460 \mathrm{C}$

Pack, A.I., Pack, A.M., Rodgman, E., Cucchiara, A., Dinges, D.F., Schwab, C.W., 1995. Characteristics of crashes attributed to the driver having fallen asleep. Accident Analysis \& Prevention 27 (6), 769-775. DOI:10.1016/0001-4575(95)00034-8

Radun, I., Ohisalo, J., Radun, J., Wahde, M., Kecklund, G., 2013. Driver fatigue and the law from the perspective of police officers and prosecutors. Transportation research part F: traffic psychology and behaviour 18, 159-167. DOI:10.1016/j.trf.2013.01.001

Sagaspe, P., Taillard, J., Bayon, V., Lagarde, E., Moore, N., Boussuge, J., Chaumet, G., Bioulac, B., Philip, P., 2010. Sleepiness, near-misses and driving accidents among a representative population of french drivers. Journal of sleep research 19 (4), 578-584. DOI: 10.1111/j.13652869.2009.00818.x 